\title{
Experiencias esquizotípicas en la adolescencia: propiedades psicométricas del Schizotypal Personality Questionnarie-Child
}

\author{
Eduardo Fonseca-Pedrero ${ }^{1,3}$, Javier Ortuño ${ }^{1}$, Mercedes Paino $^{2,3}$, Serafín Lemos-Giráldez ${ }^{2,3}$ y José Muñiz ${ }^{2,3}$ \\ 1 Departamento de Ciencias de la Educación. Universidad de La Rioja (España). \\ 2 Departamento de Psicología. Universidad de Oviedo (España). \\ 3 Centro de Investigación Biomédica en Red de Salud Mental, Madrid, CIBERS AM (España).
}

\begin{abstract}
Resumen: Las experiencias esquizotípicas son consideradas como la expresión conductual de vulnerabilidad latente a los trastornos del espectro esquizofrénico. El objetivo de esta investigación fue analizar la calidad psicométrica del Schizotypal Personality Questionnarie-Child (SPQ-C) en adolescentes no clínicos. La muestra final la formaron un total de 508 participantes, 208 varones, con una media de edad de 13.9 años $(D T=1.7)$. Los resultados mostraron que las experiencias esquizotípicas son comunes entre la población adolescente. Los modelos dimensionales hipotetizados sometidos a prueba, mediante análisis factorial confirmatorio, indicaron que el modelo tridimensional fue el que mejores índices de ajuste presentó en comparación con los modelos competentes. El coeficiente alfa de Cronbach para la puntuación total del SPQ-C fue .90. Las dimensiones del SPQ-C mostraron correlaciones estadísticamente significativas con otros autoinformes que valoraban sintomatología emocional y comportamental y estrés percibido. Ningún elemento del SPQ-C mostró funcionamiento diferencial del ítem para el factor sexo. El SPQ-C presentó un adecuado comportamiento psicométrico en esta muestra. Asimismo, la esquizotipia es un constructo de naturaleza multidimensional que puede ser evaluado de forma precisa en población adolescente con la finalidad de mejorar las estrategias de detección temprana en el campo de los trastornos mentales graves. Palabras clave: Esquizotipia; esquizotípica; validación; fiabilidad; adoles-
\end{abstract} centes; SPQ.

\begin{abstract}
Title: Schizotypal experiences in adolescence: Psychometric properties of Schizotypal Personality Questionnarie-Child

Abstract: Schizotypal experiences are considered as the behavioral expression of latent vulnerability to the schizophrenia-spectrum disorders. The main goal was to analyze the psychometric quality of Schizotypal Personality Questionnarie-Child (SPQ-C) in non-clinical adolescents. The final sample was comprised of a total of 508 participants, 208 were male, with a mean age of 13.9 years $(S D=1.7)$. The results showed that schizotypal traits are common among adolescents. Hypothesized dimensional models tested by confirmatory factor analysis, indicated the three-dimensional model was the best fit indices presented in comparison with competing models. Cronbach's alpha coefficient for the total score was .90. The dimensions of the SPQ-C showed significant correlations with self-reports that assessed emotional and behavioral problems and perceived stress. No items on SPQ-C showed differential item functioning by gender. The SPQ$\mathrm{C}$ scores showed adequate psychometric properties. Also, schizotypy is a multidimensional construct that can be accurately assessed in adolescents in order to improve early detection strategies in the field of severe mental disorders.

Key words: Schizotypy; schizotypal; validation; reliability; adolescents;
\end{abstract} SPQ.

\section{Introducción}

El estudio del fenotipo psicótico en la población general se encuentra en un momento álgido dentro del panorama científico internacional (Linscott y van Os, 2013). La psicosis es un desorden mental devastador e incapacitante que afecta al 2-3\% de la población (Perälä et al., 2007). Los costes directos e indirectos que se generan tanto a nivel personal y familiar, como a nivel sanitario y social, son cuantiosos y por ello es una de las principales líneas de actuación dentro de las políticas gubernamentales en materia de salud mental. Asimismo, la adolescencia es una época interesante para la evaluación, detección e identificación precoz de personas de riesgo de padecer un trastorno mental grave por diversos motivos, a saber: a) los signos y síntomas de psicosis comienzan en torno a 3-5 años antes de la primera hospitalización (Häfner y an Der Heiden, 1999); b) la mayoría de los casos de psicosis debutan en la adolescencia tardía o temprana adultez (van Os y Kapur, 2009); c) se evitan efectos de confundido frecuentemente encontrados en pacientes con psicosis (medicación, hospitalización); d) las experiencias psicóticas atenuadas en adolescentes predicen el desarrollo de trastornos del espectro esquizofrénico (Linscott y van Os, 2013;

* Dirección para correspondencia [Correspondence address]

Eduardo Fonseca-Pedrero. Departamento de Ciencias de la Educación. Universidad de la Rioja. C/ Luis de Ulloa, s/n, Edificio VIVES. 26002, Logroño, La Rioja (España).

E-mail: eduardo.fonseca@unirioja.es
Zammit et al., 2013); y e) es posible que una intervención temprana en los estadios iniciales del trastorno evite la transición al cuadro clínico y reduzca o mitigue el impacto del mismo a múltiples esferas.

Los síntomas de psicosis, como pudieran ser el pensamiento mágico, la anhedonia, las experiencias alucinatorias y/o la ideación delirante, se pueden encontrar presentes en la población general, por debajo del umbral clínico, y sin estar asociados necesariamente a un trastorno mental y necesidad de tratamiento. Estudios epidemiológicos indican que aproximadamente el 5-8\% de la población general informa de alguna experiencia psicotiforme (Linscott y van Os, 2013; van Os, Linscott, Myin-Germeys, Delespaul y Krabbendam, 2009). En población adolescente las tasas de prevalencia suelen ser ligeramente superiores a las encontradas en adultos llegando al 30\%, y situándose la prevalencia media entre 7.517\% (Barragan, Laurens, Navarro y Obiols, 2011; FonsecaPedrero, Santarén-Rosell, Paino y Lemos Giraldez, 2013; Kelleher et al., 2012; Wigman et al., 2011). Los modelos dimensionales de psicosis sugieren que el fenotipo psicótico se distribuye a lo largo de un continuum psicopatológico de gravedad. En su parte más extrema se situaría el cuadro clínico que se podría expresar en forma de psicosis o alguno de los trastornos del espectro esquizofrénico (p. e., trastorno esquizotípico de la personalidad). A lo largo de este continuo hipotético se podrían encontrar expresiones intermedias (subclínicas) que dependiendo de la ocurrencia, intensidad y persistencia de tales experiencias psicotiformes, así como de la comorbilidad asociada, la disfunción social y de su interac- 
ción con factores ambientales y/o genéticos, podrían conducir a un trastorno mental grave (Kaymaz et al., 2012).

Estudios longitudinales independientes señalan que los participantes sanos que informan de experiencias esquizotípicas, en comparación con aquellos que no informan de tales experiencias, tienen un mayor riesgo futuro de transitar hacia un trastorno del espectro esquizofrénico (Dominguez, Wichers, Lieb, Wittchen y van Os, 2011; Gooding, Tallent y Matts, 2005; Debbané et al., in press; Werbeloff et al., 2012; Zammit et al., 2013). No obstante, es igualmente cierto que estudios recientes señalan la baja especificidad de tales experiencias, y que su evolución no sólo se circunscribe al diagnóstico formal de psicosis sino también hacia otros trastornos mentales (p. e., depresión) (Fisher et al., 2013). Más aún, este tipo de individuos que informan de este conjunto de experiencias y rasgos también suelen presentar sutiles alteraciones emocionales, comportamentales, neurocognitivas, y/o sociales (Fonseca-Pedrero et al., 2011; Horan, Blanchard, Clark y Green, 2008; Kwapil y Barrantes-Vidal, in press; Lenzenweger, 2010; Raine, 2006), similares a las encontradas en pacientes con esquizofrenia, y que podrían ser claro indicativo de su "estado mental de riesgo". En este sentido, las experiencias esquizotipicas representarían la vulnerabilidad latente a la psicosis en la población general, y podrían considerarse como un marcador de riesgo exofenotípico para el estudio de este conjunto de síndromes (van Os et al., 2009).

Una de las líneas actuales de investigación en el campo de la psicosis se fundamenta en la idea de detectar precozmente a aquellos individuos de riesgo de psicosis mediante instrumentos psicométricos. Este hecho ha impulsado, entre otros aspectos, la construcción y validación de autoinformes para la evaluación de la esquizotipia y el fenotipo psicótico subclínico (Fonseca-Pedrero, LemosGiráldez, et al., 2011). Concretamente, en población adolescente se pueden hallar las Junior Schizotypy Scales (Rawlings y MacFarlane, 1994), el Schizotypy Traits Questionnarie for children (Cyhlarova y Claridge, 2005) o el Cuestionario Oviedo para la Evaluación de la Esquizotipia (ESQUIZO-Q) (Fonseca-Pedrero, Muñiz, Lemos-Giráldez, Paino y Villazón-García, 2010). Asimismo, diversos autoinformes inicialmente diseñados para su uso en adultos han sido administrados en adolescentes. Un ejemplo de ello es el Schizotypal Personality Questionnaire (SPQ) (Raine, 1991) o su versión abreviada (SPQ-B) (Raine y Benishay, 1995). Recientemente el grupo de Raine ha realizado una adaptación del SPQ-B a población infanto-juvenil y lo han denominado SPQ-child (SPQ-C) (Ericson, Tuvblad, Raine, Young-Wolff y Baker, 2011; Raine, Fung y Lam, 2011). La adaptación del SPQ a este sector de la población se fundamentó básicamente en mejorar la redacción y la reformulación de algunos ítems para facilitar su comprensión. El estudio de las propiedades psicométricas en muestras representativas de niños y adolescentes indicó que los niveles de consistencia interna para las subescalas oscilaban entre .60 y .81 (Ericson et al., 2011; Raine et al., 2011).
Cuando se examina la estructura factorial que subyace a este tipo de autoinformes se encuentra que la esquizotipia es un constructo de naturaleza esencialmente multidimensional. El número y contenido de las dimensiones dependen en gran medida del instrumento de medida, las técnicas estadísticas utilizadas y la muestra analizada. Concretamente, utilizando el SPQ o SPQ-B, el modelo tridimensional de Raine et al. (1994) ha sido ampliamente replicado, y se muestra consistente a través de los estudios (Fonseca-Pedrero, Paino, Lemos-Giráldez, Sierra-Baigrie y Muñiz, 2011; Fossati, Raine, Carretta, Leonardi y Maffei, 2003; Wuthrich y Bates, 2006). Dicho modelo se concreta en tres dimensiones de esquizotipia, a saber, Positiva (Cognitivo-Perceptual), Interpersonal (Negativa) y de Desorganización; no obstante, es igualmente cierto que otros estudios han encontrados dos, tres, cuatro o incluso más dimensiones (Cohen, Matthews, Najolia y Brown, 2010; Compton, Goulding, Bakeman y McClure-Tone, 2009; Fonseca-Pedrero et al., 2014). Utilizando el SPQ-C, se ha hallado que el modelo tridimensional de Raine et al. (1994) fue el que mejores índices de bondad de ajuste presentó en comparación con el resto de los modelos hipotéticos sometidos a prueba (Raine et al., 2011).

Dentro de este contexto de investigación, el propósito del presente trabajo fue examinar las propiedades psicométricas del SPQ-C (Raine et al., 2011) en una muestra de adolescentes no clínicos. Con esta finalidad, se analizan las tasas de experiencias esquizotípicas autoinformadas por los participantes, se examina la estructura interna del SPQ-C, se estima la fiabilidad de las puntuaciones, se obtienen fuentes de validez en relación con otras variables externas y se analiza el funcionamiento diferencial de los ítems en función del sexo. Este objetivo general permite conocer la calidad psicométrica del SPQ-C, en su adaptación y validación al español, en un grupo de edad de especial riesgo de psicosis, así como comprender la expresión fenotípica de la esquizotipia y su relación con diferentes variables emocionales y comportamentales.

\section{Método}

\section{Participantes}

En el estudio participaron un total de 508 estudiantes pertenecientes a diferentes centros de Educación Secundaria Obligatoria de las Comunidades Autónomas del Principado de Asturias y La Rioja. La muestra final de conveniencia estaba formada por 508 estudiantes, 208 hombres (40.9\%) pertenecientes a siete centros escolares públicos y concertados de diferentes estratos socio-económicos. La edad de los participantes osciló entré 11 y 18 años $(M=13.91 ; D T=1.71)$. La distribución final de la muestra en función de las edades fue la siguiente: 11 años $(n=31 ; 6.1 \%), 12$ años $(n=95$; $18.7 \%), 13$ años $(n=93 ; 18.3 \%), 14$ años $(n=94 ; 18.5 \%), 15$ años $(n=106 ; 20.9 \%), 16$ años $(n=55 ; 10.8 \%), 17$ años $(n=$ $20 ; 3 \%)$ y 18 años $(n=14 ; 2.8 \%)$. 


\section{Instrumentos}

Schizotypal Personality Questionnaire-Child (SPQ-C) (Raine et al., 2011). El SPQ-C es una autoinforme compuesto de 22 ítems que ha sido modificado del SPQ-B (Raine y Benishay, 1995) y que permite evaluar los rasgos esquizotípicos en población infanto-juvenil. El SPQ-C consta de tres subescalas: Cognitivo-Perceptual (ideas de referencia, suspicacia, pensamiento mágico y experiencias perceptivas extrañas), Interpersonal (ansiedad social, ausencia de amigos íntimos, afecto aplanado y suspicacia) y Desorganizada (comportamiento y lenguaje raros o excéntricos). En este estudio se utilizó un formato de respuesta Likert de cinco categorías (1= "completamente en desacuerdo"; 5 = "completamente de acuerdo"). Un formato de respuesta Likert mejora la fiabilidad de las puntuaciones y favorece la obtención de evidencias de validez (Lozano, García-Cueto, y Muñiz, 2008). Asimismo, una puntuación dimensional en psicopatología mejora sustancialmente la validez y la fiabilidad de la medición (Markon, Chmielewski y Miller, 2011).

La traducción y adaptación del instrumento de medida se llevó a cabo a través del método back-translation y siguiendo las directrices internacionales para la traducción y adaptación de tests de la International Test Commission (ITC) (Muñiz, Elosua y Hambleton, 2013). En una primera fase se llevó a cabo la traducción de los ítems por parte de dos traductores independientes y expertos en evaluación psicológica. En una segunda fase los ítems traducidos al español se retrotradujeron al inglés por dos expertos independientes y se llegó a un consenso. En una tercera fase el equipo de investigación, junto con los traductores, revisaron de forma sistemática cada uno de los ítems, obteniéndose así la versión definitiva. El SPQ-C utilizado en este estudio se presenta en el Anexo.

Strengths and Difficulties Questionnaire (SDQ) (Goodman, 1997). El SDQ es un autoinforme utilizado para la valoración de diferentes problemas emocionales y comportamentales relacionados con la salud mental en niños y adolescentes, tomando como criterio los últimos seis meses. El SDQ se compone de un total de 25 afirmaciones, que se distribuyen a lo largo de cinco subescalas (con 5 ítems cada una): Síntomas emocionales, Problemas de conducta, Hiperactividad, Problemas con los compañeros y Conducta prosocial. Las primeras cuatro subescalas forman una puntuación total de Dificultades. El formato de respuesta es Likert de tres puntos (0-2) (No; A veces; Si). En todas las subescalas, a excepción de Conducta prosocial, una mayor puntuación es indicativa de mayor sintomatología afectiva y/o comportamental. En este estudio se ha utilizado la versión adaptada y traducida al español disponible en internet (http://www.sdqinfo.com) (Fonseca-Pedrero, Paino, Lemos-Girádez y Muñiz, 2011).

Student Stress Inventory-Stress Manifestations (SSI-SM) (Fimian, Fastenau, Tashner, y Cross, 1989). El SSI-SM es un autoinforme que consta de 22 ítems que valora el estrés como respuesta en población adolescente. Se evalúan las manifestaciones de estrés percibido en tres ámbitos: emocionales
(10 ítems), fisiológicas (6 ítems) y conductuales (6 ítems). El formato de respuesta es tipo Likert de cinco puntos (0-5) (en absoluto, pocas veces, algunas veces, a menudo y totalmente). En este trabajo se utilizó la versión adaptada y validada en población española realizada por Escobar et al. (2011), que presentó adecuadas propiedades psicométricas a nivel de fiabilidad y evidencias de validez.

\section{Procedimiento}

La administración de los instrumentos de medida se llevó a cabo de forma colectiva, en grupos de 10 a 35 estudiantes, durante el horario escolar y en un aula acondicionada para este fin. El estudio fue presentado a los participantes como una investigación sobre las diversas características de la personalidad, asegurándoles la confidencialidad de sus respuestas, así como el carácter voluntario de su participación. Se obtuvo el consentimiento informado de los participantes y de los centros escolares. Los alumnos no recibieron ningún incentivo por su participación. La administración de los instrumentos de medida se realizó en todo momento bajo la supervisión de un investigador. Este estudio se enmarca dentro de una investigación más amplia sobre detección e intervención temprana en los trastornos psicológicos en la adolescencia.

\section{Análisis de datos}

En primer lugar, se calcularon los estadísticos descriptivos para los ítems del SPQ-C. En segundo lugar, se analizó la estructura interna de las puntuaciones del autoinforme mediante análisis factoriales confirmatorios para datos ordinales. El método de estimación fue WLSMV (Weighted Least Squares Mean and Variance Adjusted). Los índices de bondad de ajuste utilizados fueron: el índice de ajuste comparativo (CFI), el índice de Tucker-Lewis (TLI), el error cuadrático medio de aproximación (RMSE $A$ ) y la raíz cuadrada media residual ponderada ( $W R M R$ ). Para que exista un buen ajuste de los datos al modelo, los valores de CFI y TLI deberían ser superiores a .95 y los valores RMSE $A$ deberían ser inferiores a .08 para un ajuste razonable, e inferiores a .05 para un buen ajuste (Hu y Bentler, 1999). Se sometieron a prueba diferentes modelos dimensionales hipotetizados. Para una revisión de los modelos sometidos a prueba en este trabajo se puede consultar Fonseca-Pedrero et al. (2009).

En tercer lugar, se estimó el coeficiente alfa de Cronbach de las puntuaciones de las subescalas y la puntuación total del SPQ-C. En cuarto lugar, con la finalidad de obtener evidencias de validez en relación con otras variables, se examinaron las correlaciones de Pearson entre las subescalas del SPQ-C y dos autoinformes que valoraban sintomatología emocional y comportamental y estrés percibido. Finalmente, se examinó el funcionamiento diferencial de los ítems (DIF) en función del sexo de los participantes. Un ítem presenta DIF cuando la probabilidad de ser resuelto correctamente por individuos con el mismo nivel en el rasgo varía en fun- 
ción de su grupo de pertenencia (sexo, cultura, nivel socioeconómico) (Elosúa, 2003). En este trabajo se empleó el test generalizado de Mantel-Haenszel (GMH) (Mantel y Haenszel, 1959), concretamente the Generalized Ordinal MH statistic-QGMH(2). El procedimiento de Mantel-Haenszel se encuentra entre los más utilizados para evaluar DIF, debido a su simplicidad de cálculo e interpretación. El nivel de significación estadística se fijo en $p<.01$. Para el análisis de los datos se utilizaron los paquetes estadísticos SPSS 15.0 (Statistical Package for the Social Sciences, 2006), Mplus 5.2 (Muthén y Muthén, 1998-2007) y GHMDIF (Fidalgo, 2011).

\section{Resultados}

\section{Estadísticos descriptivos}

En la Tabla 1 se presentan los estadisticos descripitivos para los ítems del SPQ-C referidos a la media, desviación típica, asimetría y curtosis. Asimismo, se recoge el porcentaje de alumnos que selecionaron las opciones de respuestas 4 ó 5 en los 22 ítems del SPQ-C. Por ejemplo, el 3.3\% de los participantes informó estar "de acuerdo" o "totalmente de acuerdo" con la afirmación "soy poco amistoso y triste". No se encontraron diferencias estadísticamente significativas entre las puntaciones medias de la subescalas del SPQ-C en función del sexo. Las mujeres presentaron mayores puntuaciones medias totales en el SPQ-C y en la dimensión Cognitivo-Perceptual, si bien las diferencias no alcanzaron la significación estadística. La edad correlacionó de forma estadísticamente significativa con la dimensión Desorganizada $(r=.12 ; p<.01)$ y la dimensión Interpersonal $(r=.10 ; p<.05)$ del SPQ-C.

Tabla 1. Estadísticos descriptivos de los ítems del Schizotypal Personality Questionnarie-Child.

\begin{tabular}{lccccc}
\hline Items & $M$ & $D T$ & Asimetría & Curtosis & \% respuestas afirmativas* \\
\hline 1 & 1.45 & 0.83 & 2.06 & 4.17 & 3.3 \\
2 & 2.04 & 1.3 & 0.92 & -0.49 & 18.3 \\
3 & 2.12 & 1.19 & 0.82 & -0.3 & 14.6 \\
4 & 1.74 & 1.17 & 1.42 & 0.74 & 13 \\
5 & 1.93 & 1.25 & 1.08 & -0.12 & 16.4 \\
6 & 1.57 & 1.03 & 1.91 & 2.91 & 7.1 \\
7 & 1.79 & 1.17 & 1.37 & 0.79 & 11.8 \\
8 & 2 & 1.2 & 0.95 & -0.23 & 14.3 \\
9 & 1.88 & 1.23 & 1.22 & 0.31 & 13.4 \\
10 & 1.83 & 1.2 & 1.15 & -0.05 & 14.8 \\
11 & 2.24 & 1.29 & 0.64 & -0.82 & 19.9 \\
12 & 1.82 & 1.29 & 1.32 & 0.34 & 15.2 \\
13 & 1.78 & 1.07 & 1.2 & 0.39 & 10 \\
14 & 2.26 & 1.31 & 0.63 & -0.79 & 18.3 \\
15 & 2.05 & 1.17 & 0.8 & -0.41 & 13.4 \\
16 & 2.12 & 1.29 & 0.79 & -0.62 & 17.7 \\
17 & 2.15 & 1.4 & 0.86 & -0.64 & 21.1 \\
18 & 1.8 & 1.17 & 1.4 & 0.94 & 10.8 \\
19 & 1.54 & 1.01 & 1.96 & 3.01 & 7.3 \\
20 & 1.88 & 1.2 & 1.17 & 0.21 & 13 \\
21 & 2.33 & 1.27 & 0.49 & -0.93 & 20.5 \\
22 & 2.52 & 1.49 & 0.44 & -1.27 & 29.9 \\
\hline & & & & &
\end{tabular}

* Porcentaje de participantes que seleccionaron las opciones de respuesta 4 ó 5 en los ítems del SPQ-C.

\section{Evidencias de estructura interna}

Se sometieron a prueba cuatro modelos dimensionales hipotéticos de esquizotipia. El primer modelo consideraba la presencia de una única dimensión general de esquizotipia que podría explicar toda la sintomatología subyacente. El segundo modelo proponía dos dimensiones generales de esquizotipia, una de síntomas positivos y otra de síntomas negativos. El tercer modelo, el propuesto por Raine et al. (1994), postulaba la presencia de tres dimensiones (Cognitivo-Perceptual, Interpersonal y Desorganizada), en donde los ítems 7, 9, 14 y 17 saturaban en las dimensiones CognitivoPerceptual e Interpersonal. Finalmente, un cuarto modelo propuesto por Raine y Benishay (1995), en donde los ítems 9 y 17 pertenecían a la dimensión Cognitivo Perceptual y los ítems 7 y 14 a la dimensión Interpersonal. Los índices de bondad de ajuste para los modelos hipotetizados sometidos a prueba se presentan en la Tabla 2. Como se puede observar, el modelo que mejores índices de bondad de ajuste presentó fue el modelo tridimensional de Raine et al. (1994). Las cargas factoriales estandarizadas para este modelo se presentan en la Tabla 3. Las correlaciones entre las dimensiones del SPQ-C oscilaron entre .60 (FI-FII) y .79 (FI-FIII) $(p<.01)$.

Tabla 2. Índices de bondad de ajuste para los modelos dimensionales sometidos a prueba mediante análisis factorial confirmatorio.

\begin{tabular}{lcccccc}
\hline Modelos & $\chi^{2}$ & $g l$ & CFI & TLI & RMSEA & WRMR \\
\hline Unidimensional & 597.3 & 84 & .81 & .92 & 0.11 & 1.67 \\
Bidimensional & 531.6 & 85 & .84 & .93 & 0.10 & 1.55 \\
$\begin{array}{l}\text { Tridimensional } \\
\text { Raine et al. (1994) }\end{array}$ & 452.60 & 88 & .87 & .95 & 0.09 & 1.39 \\
$\begin{array}{l}\text { Tridimensional } \\
\text { Raine et al. (1995) }\end{array}$ & 397.2 & 91 & .89 & .96 & 0.08 & 1.25 \\
\hline
\end{tabular}

Nota: $\chi^{2}=$ Chi cuadrado; $g l=$ grados de libertad; CFI = índice de ajuste comparativo; TLI = índice de Tucker-Lewis (TLI); RMSE $A=$ error cuadrático medio de aproximación; $W R M R=$ raíz cuadrada media residual ponderada.

\section{Estimación de la consistencia interna de las puntuaciones}

El coeficiente alfa de Cronbach para la puntuación total del SPQ-C fue de .90, para la dimensión CognitivoPerceptual fue .86, para la dimensión Interpersonal fue $.83 \mathrm{y}$ para la dimensión Desorganizada fue .80. Todos los índices de discriminación, tanto para las subescalas tomadas de forma individual como para la puntuación total del instrumento de medida, fueron superiores a .30 .

\section{Evidencias de validez en relación con variables externas}

En la Tabla 4 se presentan las correlaciones de Pearson entre las subescalas de los diferentes instrumentos de medi- 
da utilizados. Como se puede observar, la mayoría de las correlaciones fueron estadísticamente significativas en sentido positivo, a excepción de la relación entre las subescalas del SPQ-C y la subescala Conducta Prosocial del SDQ. Véase que las correlaciones oscilaron entre -.14 (SPQ-C Desorganizada y SDQ Prosocial) y .48 (SPQ-C Desorganizada y SSISM Emocional).

Análisis del funcionamiento diferencial de los ítems del SPQ-C en función del sexo de los participantes

Los resultados indicaron que ningun ítem del SPQ-C presentó un funcionamiento diferencial en la variable sexo.

\section{Discusión y conclusiones}

El objetivo principal fue examinar la calidad métrica del Schizotypal Personality Questionarie-Child (SPQ-C) (Raine et al., 2011) en una muestra incidental de adolescentes españoles. Con esta finalidad, se analizaron las tasas de experiencias esquizotípicas autoinformadas, se examinó la estructura interna del SPQ-C, se estimó la fiabilidad de las puntuaciones y se obtuvieron diferentes evidencias de validez. Este objetivo permite conocer las propiedades psicométricas del SPQ-C para su uso como instrumento de cribado breve y sencillo en un grupo de edad de especial riesgo de desarrollar un trastorno mental. Del mismo modo, este objetivo pretende arrojar nuevas evidencias empíricas sobre la distribución y la expresión de las experiencias y rasgos esquizotípicos en población juvenil, así como conocer sus nexos de unión con variables emocionales y comportamentales a nivel subclínico.
En términos generales, los resultados parecen indicar que las puntuaciones de SPQ-C presentaron un adecuado comportamiento psicométrico y que este instrumento de medida podría ser utilizado para la valoración de las experiencias y los rasgos de esquizotipia en población adolescente.

Tabla 3. Cargas factoriales completamente estandarizadas para el modelo tridimensional de Raine et al. (1994).

\begin{tabular}{|c|c|c|c|}
\hline Ítems & F I & F II & F III \\
\hline 1 & & .53 & \\
\hline 2 & .67 & & \\
\hline 3 & & & .74 \\
\hline 4 & .70 & & \\
\hline 5 & .71 & & \\
\hline 6 & & & .72 \\
\hline 7 & .43 & .36 & \\
\hline 8 & & & .56 \\
\hline 9 & .44 & .27 & \\
\hline 10 & .78 & & \\
\hline 11 & & .73 & \\
\hline 12 & .59 & & \\
\hline 13 & & & .68 \\
\hline 14 & .17 & .52 & \\
\hline 15 & & .75 & \\
\hline 16 & .71 & & \\
\hline 17 & .31 & .38 & \\
\hline 18 & & .69 & \\
\hline 19 & & & .85 \\
\hline 20 & & & .77 \\
\hline 21 & & .74 & \\
\hline 22 & & .53 & \\
\hline
\end{tabular}

Nota: todas las cargas factoriales estimadas fueron estadísticamente significativas $(p<.01)$

Tabla 4. Correlaciones de Pearson entre las subescalas del Schizotypal Personality Questionnarie-Child (SPQ-C) y las del Strengths and Difficulties Questionnaire (SDQ) y el Student Stress Inventory-Stress Manifestations (SSI-SM).

\begin{tabular}{|c|c|c|c|c|c|c|c|c|}
\hline SPQ-C & $\begin{array}{c}\text { SDQ } \\
\text { Emocional }\end{array}$ & $\begin{array}{c}\text { SDQ } \\
\text { Problemas } \\
\text { de conducta }\end{array}$ & $\begin{array}{c}\text { SDQ } \\
\text { Problemas con } \\
\text { los compañeros }\end{array}$ & $\begin{array}{c}\text { SDQ } \\
\text { Hiperactividad }\end{array}$ & $\begin{array}{c}\text { SDQ } \\
\text { Prosocial }\end{array}$ & $\begin{array}{c}\text { SSI } \\
\text { Emocional }\end{array}$ & $\begin{array}{c}\text { SSI } \\
\text { Conductual }\end{array}$ & $\begin{array}{c}\text { SSI } \\
\text { Fisiológico }\end{array}$ \\
\hline Cognitivo-Perceptual & $.476^{* *}$ & $.247 * *$ & $.354 * *$ & $.210^{* *}$ & -.041 & $.400^{* *}$ & $.253 * *$ & $.174 * *$ \\
\hline Interpersonal & $.464 * *$ & $.234 * *$ & $.419 * *$ & $.097^{*}$ & $-.104 *$ & $.360 * *$ & $.155^{* *}$ & $.156^{* *}$ \\
\hline Desorganizado & $.450 * *$ & $.321 * *$ & $.352 * *$ & $.226 * *$ & $-.143^{* *}$ & $.470 * *$ & $.250 * *$ & $.272 * *$ \\
\hline
\end{tabular}

$* * p<01 ; * p<$

Los resultados mostraron que los rasgos y las experiencias esquizotípicas son comunes entre la población adolescente. Entre el 3.3 y el $29.9 \%$ de la muestra analizada presentaría alguna experiencia esquizotípica. Las tasas de rasgos y experiencias psicotiformes encontradas son similares a las halladas en investigaciones previas, tanto en adolescentes como en adultos no clínicos (Fonseca-Pedrero et al., 2011; Linscott y van Os, 2013; van Os et al., 2009; Wigman et al., 2011). Por ejemplo, Fonseca-Pedrero et al. (2011), utilizando una muestra de 1438 adolescentes españoles, encontraron que aproximadamente el $43 \%$ de los participantes informó de algún síntoma relacionado con el pensamiento mágico, la ideación referencial, las experiencias delirantes y/o las pseudo-alucinaciones. Por su parte, un re- ciente meta-análisis indica que la prevalencia media de las experiencias psicóticas atenuadas en población infantojuvenil se sitúa entre 7.5-17\% (Kelleher et al., 2012). Estos datos señalan que las experiencias esquizotípicas se pueden encontrar presentes en la población general adolescente extendiéndose más allá de las fronteras del fenotipo clínico (van Os et al., 2009). Un aspecto digno de mención es que la esquizotipia, como constructo, recoge un mayor rango de muestras de comportamiento del fenotipo psicótico (Cognitivo-Perceptual, Interpersonal y Desorganizada), en comparación con el constructo de síntomas psicóticos atenuados, que suele recoger aspectos más relacionados con la dimensión positiva de la esquizotipia. 
Las puntuaciones del SPQ-C presentaron un correcto comportamiento psicométrico. La consistencia interna para las subescalas y la puntuación total del instrumento de medida fueron superiores a .80. Estos datos son claramente convergentes con estudios previos (Ericson et al., 2011; Raine et al., 2011). Se debe mencionar que la posible mejora de la fiabilidad de las puntuaciones del SPQ-C podría venir determinada por el formato de respuesta tipo Likert de 5 puntos utilizada en este trabajo. En la literatura especializada se aconseja la utilizacion de este formato de respuesta para mejorar la calidad métrica de las puntuaciones (Lozano et al., 2008), así como para la construcción de puntuaciones dimensionales en medidas psicopatológicas (Markon et al., 2011). Por otro lado, ningún item presentó un funcionamiento diferencial en función del sexo de los participantes, aspecto que garantiza la comparación y la equidad de las puntuaciones entre los grupos a comparar.

El análisis de la estructura dimensional subyacente a los ítems del SPQ-C indicó que el modelo tridimensional de Raine et al. (1994) concretado en las facetas CognitivoPerceptual, Interpersonal y de Desorganización, fue el que mejores índices de bondad de ajuste presentó en comparación con el resto de modelos propuestos. Estos resultados son convergentes con estudios realizados previamente en población adolescente y adulta (Fonseca-Pedrero et al., 2014; Fonseca-Pedrero, Paino, et al., 2011; Fossati et al., 2003; Wuthrich y Bates, 2006) y resaltan el paralelismo fenotípico con los encontrado en pacientes con esquizofrenia. Por ejemplo, Fonseca-Pedrero et al. (2009), utilizando el SPQ-B en una muestra representativa de adolescentes españoles, encontraron que el modelo tridimensional de Raine et al. (1994), fue el que mejores índices de bondad de ajuste mostró. A resultados similares llegaron Raine et al. (2011) cuando comprobaron la estructura del SPQ-C en una muestra representativa de adolescentes no clínicos. Estos datos apuntan hacia una estructura tripartita de la esquizotipia, si bien es cierto que puede variar en función del instrumento de medida, de la muestra y de la técnica estadística utilizada (Cohen et al., 2010; Compton et al., 2009).

Las puntuaciones del SPQ-C mostraron asociaciones estadísticamente significativas con síntomas emocionales y comportamentales y con estrés percibido. Estos datos son convergentes con los obtenidos en estudios previos, tanto en población general de adultos como en adolescentes no clínicos (Fonseca-Pedrero, Paino, Lemos-Giráldez et al., 2011; Horan et al., 2008; Kwapil et al., 2008; Lenzenweger, 2010; Raine, 2006; Wigman et al., 2011). Por ejemplo, Wigman et al. (2011), utilizando la Community Assessment Psychic Experiences-42 y el SDQ en una muestra de adolescentes, encontraron que aquellos que informaron de experiencias psicóticas atenuadas también presentaban una mayor sintomatología emocional y comportamental. A partir de estos resultados son dignos de mención dos aspectos. Por un lado, y de acuerdo con los modelos dimensionales de psicosis, las experiencias y rasgos esquizotípicos podrían concebirse como una variación dentro del continuo de fenotipo psicótico, cuantitativamente menos grave y cualitativamente similar a la sintomatología encontrada en pacientes con psicosis. Por otro, apresar estas variables podría tener relevancia desde un punto de vista clínico e investigador y apoyar los modelos de diátesis-estrés. La combinación entre la vulnerabilidad que supone la puntuación extrema en esquizotipia -evaluada por el SPQ-C- y un elevado estrés percibido podría producir como consecuencia una mayor sintomatología tanto emocional como comportamental y, por lo tanto, aumentar la probabilidad de transitar hacía un trastorno mental serio. Es posible que los problemas emocionales y de conducta, unidos al estrés percibido en los adolescentes que informan también de experiencias y rasgos esquizotípicos, funcionen como factores moderadores y puedan aumentar de manera aditiva o sinérgica el riesgo de psicosis (Linscott y van Os, 2013).

Los resultados encontrados en el presente estudio deberían interpretarse a la luz de las siguientes limitaciones. En primer lugar, la adolescencia es una época donde la personalidad se encuentra aún en desarrollo por lo que los resultados se deben enmarcar dentro de los cambios madurativos que ocurren en esta etapa. En segundo lugar, existe la problemática inherente a la aplicación de cualquier tipo de autoinforme, con el consabido efecto de estigmatización, la posible falta de comprensión de ciertos ítems o la escasa capacidad de introspección por parte de los estudiantes, por lo que hubiese sido interesante la utilización de informantes externos, vía heteroinforme o entrevistas estructuradas. En tercer lugar, no se utilizó una escala de infrecuencia de respuesta útil para detectar patrones de respuestas azarosos o pseudoazarosos. En cuarto lugar, se debe tener en cuenta que en este estudio se ha utilizado un formato de respuesta tipo $\mathrm{Li}$ kert de cinco puntos, si bien el SPQ-C en su versión original utiliza un formato de respuesta dicotómico. Este hecho se debe tener presente a la hora de la generalización de los resultados a otras muestras de interés. Finalmente no se debe perder de vista que estas experiencias son bastante comunes en este sector de la población, y no necesariamente tienen que concebirse como un marcador específico para los trastornos del espectro esquizofrénico. Es necesario considerar otras variables que informen acerca de su significado clínicopatológico, como por ejemplo, la persistencia, la intensidad, el malestar asociado o el impacto en el funcionamiento social/laboral/escolar, y siempre dentro de un modelo biopsicosocial más holístico que recoja la compleja interacción que se establece entre factores genéticos y ambientales.

Estos resultados arrojan nuevas pistas que permiten mejorar la comprensión de los rasgos y experiencias esquizotípicas en este sector de la población. El paradigma de "alto riesgo psicométrico" podría ser una estrategia útil y válida para la detección de participantes con vulnerabilidad a los trastornos mentales graves en contextos educativos y/o asistenciales. Asimismo, estos resultados enfatizan, sin lugar a dudas, la necesidad de valorar tempranamente tales rasgos y experiencias en población infanto-juvenil, con la finalidad de tratar de evitar la posible transición hacia un cuadro clínico. 
En futuros estudios sería interesante establecer puntos de corte específicos, así como seguir longitudinalmente a aquellos participantes con puntuaciones elevadas en este tipo de autoinformes, de cara a la obtención de índices de sensibilidad y especificidad. La utilización combinada de otros factores de riesgo (por ejemplo, atención sostenida, marcadores biológicos), de cara a mejorar la predicción en las tasas de

\section{Referencias}

Barragan, M., Laurens, K. R., Navarro, J. B. y Obiols, J. E. (2011). Psychotic-like experiences and depressive symptoms in a community sample of adolescents. European Psychiatry, 26, 396-401.

Cohen, A. S., Matthews, R. A., Najolia, G. M. y Brown, L. A. (2010). Toward a more psychometrically sound brief measure of schizotypal traits: introducing the SPQ-Brief Revised. Journal of Personality Disorders, 24, 516-537.

Compton, M. T., Goulding, S. M., Bakeman, R. y McClure-Tone, E. B. (2009). Confirmation of a four-factor structure of the Schizotypal Personality Questionnaire among undergraduate students. Schizophrenia Research, 111, 46-52.

Cyhlarova, E. y Claridge, G. (2005). Development of a version of the Schizotypy Traits Questionnaire (STA) for screening children. Schizophrenia Research, 80(2-3), 253-261.

Debbané, M., Eliez, S., Badoud, D., Conus, P., Flückiger, R., y SchultzeLutter, F. (in press). Developing psychosis and its risk states through the lens of schizotypy. Schizophrenia Bulletin.

Dominguez, M. G., Wichers, M., Lieb, R., Wittchen, H.-U. y van Os, J. (2011). Evidence that onset of clinical psychosis is an outcome of progressively more persistent subclinical psychotic experiences: An 8Year Cohort Study. Schizophrenia Bulletin, 37, 84-93.

Elosúa, P. (2003). Sobre la validez de los tests [About test validity]. Psicothema, 15, 315-321.

Ericson, M., Tuvblad, C., Raine, A., Young-Wolff, K. y Baker, L. A. (2011). Heritability and longitudinal stability of schizotypal traits during adolescence. Behavior Genetics 41, 499-511.

Escobar, M., Blanca, M. J., Fernández-Baena, F. J. y Trianes, M. V. (2011). Adaptación española de la escala de manifestaciones de estrés del Student Stress Inventory (SSI-SM) [Spanish adaptation of the Stress Manifestations Scale of the Student Stress Inventory (SSI-SM)]. Psicothema, 23, 475-485.

Fidalgo, A. M. (2011). GMHDIF: A computer program for detecting DIF in dichotomous and polytomous items using generalized Mantel-Haenszel Statistics. Applied Psychological Measurement, 35, 247-249.

Fimian, M. J., Fastenau, P. A., Tashner, J. H. y Cross, A. H. (1989). The measure of classroom stress and burnout among gifted and talented students. Psychology in the Schools, 26, 139-153.

Fisher, H. L., Caspi, A., Poulton, R., Meier, M. H., Houts, R., Harrington, H., Arseneault, L. y Moffitt, T. E. (2013). Specificity of childhood psychotic symptoms for predicting schizophrenia by 38 years of age: a birth cohort study. Psychological Medicine, 43, 2077-2086.

Fonseca-Pedrero, E., Fumero, A., Paino, M., de Miguel, A., Ortuño-Sierra, J., Lemos Giraldez, S. y Muñiz, J. (2014). Schizotypal Personality Questionnaire: New sources of validity evidence in college students. Psychiatry Research, 219, 214-220.

Fonseca-Pedrero, E., Lemos-Giráldez, S., Paino, M., Santarén-Rosell, M., Sierra-Baigrie, S. y Ordóñez-Camblor, N. (2011). Instrumentos de medida para la evaluación del fenotipo psicótico. Papeles del Psicólogo, 32, 129-151.

Fonseca-Pedrero, E., Muñiz, J., Lemos-Giráldez, S., Paino, M. y VillazónGarcía, U. (2010). ESQUIZO-Q: Cuestionario Oviedo para la Evaluación de la Esquizotipia. Madrid: TEA ediciones.

Fonseca-Pedrero, E., Paino-Piñeiro, M., Lemos-Giráldez, S., VillazónGarcía, Ú. y Muñiz, J. (2009). Validation of the Schizotypal Personality Questionnaire-Brief Form in adolescents. Schizophrenia Research, 111, 5360 . conversión y así reducir los falsos positivos, es también una línea de investigación futura interesante.

Agradecimientos.- Esta investigación ha sido financiada por el Ministerio de Ciencia e Innovación de España (MICINN), por el Instituto Carlos III, Centro de Investigación Biomédica en Red de Salud Mental (CIBERSAM). Referencias de los proyectos: PSI 2011-28638 y PSI 2011-23818.

Fonseca-Pedrero, E., Paino, M., Lemos-Girádez, S. y Muñiz, J. (2011). Prevalencia de la sintomatología emocional y comportamental en adolescentes españoles a través del Strengths and Difficulties Questionnaire (SDQ). Revista de Psicopatología y Psicología clínica, 16, 15-25.

Fonseca-Pedrero, E., Paino, M., Lemos-Giráldez, S., Sierra-Baigrie, S. y Muñiz, J. (2011). Measurement invariance of the Schizotypal Personality Questionnaire-Brief across gender and age. Psychiatry Research, 190, 309315.

Fonseca-Pedrero, E., Paino, M., Lemos-Giráldez, S., Sierra-Baigrie, S. Ordoñez-Camblor, N. y Muñiz, J. (2011). Early psychopathological features in Spanish adolescents. Psicothema, 23, 87-93.

Fonseca-Pedrero, E., Santarén-Rosell, M., Lemos-Giráldez, S., Paino, M. Sierra-Baigrie, S. y Muñiz, J. (2011). Síntomas psicóticos subclínicos en población general adolescente [Psychotic-like experiences in the adolescent general population]. Actas Españolas de Psiquiatría, 39, 155162.

Fonseca-Pedrero, E., Santarén-Rosell, M., Paino, M.,y Lemos Giraldez, S. (2013). Cluster A maladaptive personality patterns in a non-clinical adolescent population. Psicothema, 25, 171-178.

Fossati, A., Raine, A., Carretta, I., Leonardi, B. y Maffei, C. (2003). The three-factor model of schizotypal personality: Invariance across age and gender. Personality and Individual Differences, 35, 1007-1019.

Gooding, D. C., Tallent, K. A. y Matts, C. W. (2005). Clinical status of atrisk individuals 5 years later: Further validation of the psychometric high-risk strategy. Journal of Abnormal Psychology, 114(1), 170-175.

Goodman, R. (1997). The strengths and difficuties questionnaire: a research note. Journal of Child Psychology and Psychiatry, 38, 581-586.

Häfner, H. y an Der Heiden, W. (1999). The course of schizophrenia in the light of modern follow-up studies: The ABC and WHO studies. European Archives of Psychiatry and Clinical Neuroscience, 249(SUPPL. 4), IV14-IV26.

Horan, W. P., Blanchard, J. J., Clark, L. A. y Green, M. F. (2008). Affective traits in schizophrenia and schizotypy. Schizophrenia Bulletin, 34, 856-874

Hu, L. T. y Bentler, P. M. (1999). Cut off criteria for fit indexes in covariance structure analysis: Conventional criteria versus new alternatives. Structural Equation Modeling, 6, 1-55.

Kaymaz, N., Drukker, M., Lieb, R., Wittchen, H. U., Werbeloff, N., Weiser, M., Lataster, T. y van Os, J. (2012). Do subthreshold psychotic experiences predict clinical outcomes in unselected non-help-seeking population-based samples? A systematic review and meta-analysis, enriched with new results. Psychological Medicine, 20, 1-15.

Kelleher, I., Connor, D., Clarke, M. C., Devlin, N., Harley, M. y Cannon, M. (2012). Prevalence of psychotic symptoms in childhood and adolescence: a systematic review and meta-analysis of population-based studies. Psychological Medicine, 9, 1-7.

Kwapil, T. R., \& Barrantes-Vidal, N. (in press). Schizotypy: Looking back and moving forward. Schizopbrenia Bulletin.

Lenzenweger, M. E. (2010). Schizotypy and schizophrenia: The view from experimental psychopathology. New York: Guilford Press.

Linscott, R. J. y van Os, J. (2013). An updated and conservative systematic review and meta-analysis of epidemiological evidence on psychotic experiences in children and adults: on the pathway from proneness to persistence to dimensional expression across mental disorders. Psychological Medicine, 43, 1133-1149.

Lozano, L. M., García-Cueto, E. y Muñiz, J. (2008). Effect of the number of response categories on the reliability and validity of rating scales. Methodology, 4, 73-79. 
Mantel, N. y Haenszel, W. (1959). Statistical aspects of the analysis of data from retrospective studies of disease. Journal of the National Cancer Institute, 22, 719-748.

Markon, K. E., Chmielewski, M., y Miller, C. J. (2011). The reliability and validity of discrete and continuous measures of psychopathology: a quantitative review. Psychological Bulletin, 137, 856-879.

Muñiz, J., Elosua, P. y Hambleton, R. K. (2013). Directrices para la traducción y adaptación de los tests: segunda edición [International Test Commission Guidelines for test translation and adaptation: Second edition]. Psicothema, 25, 151-157.

Muthén, L. K. y Muthén, B. O. (1998-2007). Mplus User's Guide. (Fifth ed.). Los Angeles, CA: Muthén \& Muthén.

Perälä, J., Suvisaari, J., Saarni, S. I., Kuoppasalmi, K., Isometsä, E., Pirkola, S., ... Lönnqvist, J. (2007). Lifetime prevalence of psychotic and bipolar I disorders in a general population. Archives of General Psychiatry, 64, 1928.

Raine, A. (1991). The SPQ: A scale for the assessment of schizotypal personality based on DSM-III-R criteria. Schizophrenia Bulletin, 17, 555564.

Raine, A. (2006). Schizotypal personality: Neurodevelopmental and psychosocial trajectories. Annual Review of Clinical Psychology, 2, 291-326.

Raine, A. y Benishay, D. (1995). The SPQ-B: a brief screening instrument for schizotypal personality disorder. Journal of Personality Disorders, 9, 346355.

Raine, A., Fung, A. L. y Lam, B. Y. (2011). Peer victimization partially mediates the schizotypy-aggression relationship in children and adolescents. Schizophrenia Bulletin, 37, 937-945.

Raine, A., Reynolds, C., Lencz, T., Scerbo, A., Triphon, N. y Kim, D. (1994). Cognitive-perceptual, interpersonal, and disorganized features of schizotypal personality. Schizophrenia bulletin, 20, 191-201.
Rawlings, D. y MacFarlane, C. (1994). A multidimensional schizotypal traits questionnaire for young adolescents. Personality and Individual Differences, 17(4), 489-496.

Statistical Package for the Social Sciences (2006). SPSS Base 15.0 User's Guide. Chicago, IL: SPSS Inc.

van Os, J. y Kapur, S. (2009). Schizophrenia. Lancet, 374, 635-645.

van Os, J., Linscott, R. J., Myin-Germeys, I., Delespaul, P. y Krabbendam, L. (2009). A systematic review and meta-analysis of the psychosis continuum: Evidence for a psychosis proneness-persistence-impairment model of psychotic disorder. Psychological Medicine, 39, 179-195.

Werbeloff, N., Drukker, M., Dohrenwend, B. P., Levav, I., Yoffe, R., van Os, J., Davidson, M. y Weiser, M. (2012). Self-reported attenuated psychotic symptoms as forerunners of severe mental disorders later in life. Archives of General Psychiatry, 69, 467-475.

Wigman, J. T., Vollebergh, W. A., Raaijmakers, Q. A., Iedema, J., van Dorsselaer, S., Ormel, J., Verhulst, F. C. y van Os, J. (2011). The structure of the extended psychosis phenotype in early adolescence--A cross-sample replication. Schizophrenia Bulletin, 37, 850-860.

Wuthrich, V. y Bates, T. C. (2006). Confirmatory factor analysis of the threefactor structure of the schizotypal personality questionnaire and Chapman schizotypy scales. Journal of Personality Assessment, 87, 292-304.

Zammit, S., Kounali, D., Cannon, M., David, A. S., Gunnell, D., Heron, J., Jones, P. B., Lewis, S., Sullivan, S., Wolke, D. y Lewis, G. (2013). Psychotic Experiences and Psychotic Disorders at Age 18 in Relation to Psychotic Experiences at Age 12 in a Longitudinal Population-Based Cohort Study. American Journal of Psychiatry, 170, 742-750.

(Articulo recibido: 25-01-2013; Revisado: 15-05-2013; Aceptado: 03-07-2014)

Anexo 1. Ítems que conforman la versión adaptada y validada al español del Schizotypal Personality Questionnarie-Child.

1. Soy poco amistoso y frío.

2. A veces he tenido la sensación de que había una persona o un fantasma a mi lado, aunque allí no había nadie.

3. A veces actúo de manera extraña.

4. A veces pienso que otras personas pueden leer mi mente.

5. A veces encuentro que objetos o sucesos corrientes tienen un mensaje especial para mí.

6. Soy una persona rara (extraña).

7. Siento que tengo que estar en guardia, incluso con los amigos.

8. A veces divago cuando estoy hablando.

9. Con frecuencia capto amenazas ocultas o desprecios en lo que dice o hace la gente.

10. Tengo la sensación de que otras personas me observan cuando salgo a jugar o a comprar.

11. Me siento muy incómodo en situaciones sociales con gente que no conozco.

12. He tenido experiencias como ver platillos volantes o saber que algo va a suceder antes de que ocurra.

13. A veces uso palabras de manera inusual (de forma extraña).

14. Encuentro que es mejor no dejar que otras personas sepan demasiado de mí.

15. Con frecuencia me mantengo en segundo plano en situaciones sociales.

16. A veces me siento distraído por sonidos lejanos de los que normalmente no me doy cuenta.

17. Con frecuencia tengo que impedir que la gente se aproveche de mí.

18. Me resulta difícil hacer amigos íntimos.

19. Soy una persona extraña, inusual.

20. Encuentro difícil hacer que la gente entienda lo que estoy diciendo.

21. Me siento incómodo al hablar con gente que no conozco bien.

22. Tiendo a guardar mis sentimientos para mí mismo. 